\title{
Efficacy of adalimumab in patients with crohn's disease and failure to infliximab therapy: a clinical series
}

\author{
Patricia Cordero-Ruiz, C Castro-Márquez, V Méndez-Rufián, L Castro-Laria, A Caunedo-Álvarez, \\ J Romero-Vázquez, and JM Herrerías-Gutiérrez
}

Unit of Inflammatory Bowel Diasease. Departament of Gastroenterology. Hospital Universitario Virgen Macarena. Seville, Spain

\begin{abstract}
Background: adalimumab, a human anti-TNF, is an effective induction and maintenance therapy for patients with moderate to severe Crohn s disease. It seems to be effective in patients with resistance to Infliximab, too, though the experience is more limited.

Aim: to evaluate the efficacy of adalimumab, in patients with Crohn s disease (CD) and failure to previous treatment with infliximab.

B twenty-five patients with $\mathrm{CD}$ and failure to previous treatment with infliximab were enrolled; they were treated with 160/80 (24 patients) and 80/40 (1 patient) induction doses. We analyze clinical response to treatment with adalimumab by the Crohn's disease Activity Index (CDAI) and plasma concentration of C-reactive protein (CRP), steroid sparing and complete fistula closure at week 48 .

Results: eighteen out of twenty-five patients (72\%) achieved clinical remission (CDAI score < 150) at week 24 y 15/25 (60\%) patients at week 48 . There was a statistically significant difference $(p<0.01)$ in CRP serum levels from 21 to $8 \mathrm{mg} / \mathrm{dl}$ at week 48 . Nine out of fifteen patients (60\%) treated with corticosteroids were able to discontinue steroids. Three out of eleven patients (27\%) with fistulizing Crohn s disease had complete fistula closure after the treatment. Seventy two percent of the patients $(18 / 25)$ needed to increase adalimumab to weekly dose, in order to maintain clinical response. Five out of twenty-five patients (20\%) had adverse events; two of them (8\%) with serious adverse events (tuberculous meningitis and abdominal abscess) that forced the withdrawal of treatment.

Conclusions: according to these data, adalimumab provides a clinical and analytical improvement in patients with $C D$ and failure to previous therapy with infliximab.
\end{abstract}

Key words: Adalimumab. Crohn's disease. Infliximab. Loss of response. Intolerance.

Received: 22-02-10.

Accepted: 04-02-11.

Correspondence: Patricia Cordero Ruiz. Secretaría Servicio Digestivo (8. ${ }^{a}$ planta). Hospital Universitario Virgen Macarena. Avda. Dr. Fedriani, s/n. 41009 Sevilla.

e-mail: rioga_patri@hotmail.com
Cayuela A, Rodríguez-Domínguez S, Garzón Benavides M, Pizarro-Moreno A, Giráldez-Gallego A, Cordero Fernández C. Study of mortality from colorectal cancer in the andalusian population. Rev Esp Enferm Dig 2011; 103: 294-298.

\section{INTRODUCTION}

Crohn's disease (CD) is a chronic inflammatory disorder of the gastrointestinal tract, with periods of exacerbations and remission. Although there is not only one established cause, certain environmental factors appear to be involved in genetically susceptible hosts, triggering an excessive immunological response and damage in the intestinal tissues. In this context, a great amount of pro-inflammatory cytokines, -interferon, several interleukins and tumor necrosis factor (TNF-) are released. TNFplays an essential role in $C D$ pathogenesis, thus it is the main therapeutic target.

Infliximab (IFX) was the first chimerical monoclonal antibody (IgG1) against tumor necrosis factor alpha (TNF) introduced into clinical practice and it has proven to be effective in remission induction and maintenance in patients with $\mathrm{CD}$ from moderate to severe and in fistulizing disease. IFX is $75 \%$ human sequences and $25 \%$ mice sequences and has immunogenic potential which may be responsible for reduced efficacy and systemic hypersensitive reactions or at the point of infusion $(1,2)$. Adalimumab (ADA) is a monoclonal antibody with high affinity and specificity against TNF, thus it may be considered an adequate therapeutic option in patients with inflammatory bowel disease (IBD) who are intolerant or unresponsive to IFX treatment. Both drugs are comprised within the same therapeutic line and share similar indications, although ADA is a 
humanized antibody. This molecule has shown positive clinical effects in patients with rheumatologic diseases (2) and recent studies also show its effectiveness in CD cases with intolerance or loss of responsiveness to IFX $(3,4)$. The CLASSIC I study showed that $160 \mathrm{mg}$ doses of subcutaneous ADA, followed by $80 \mathrm{mg}$ two weeks later were significantly more effective than placebo in the induction of remission in 299 patients with moderate-severe CD (5). More recently, the CLASSIC II study proved that $40 \mathrm{mg}$ of subcutaneous ADA every week or two was more effective than placebo in the maintenance of remission after 56 weeks in 55 patients with moderate-severe $\mathrm{CD}$ who had achieved remission after ADA induction therapy (6).

The aim of our study was to assess efficacy and safety of ADA in patients with previous failure to IFX in the management of $\mathrm{CD}$.

\section{MATERIAL AND METHODS}

Twenty-five patients with diagnosed CD based on endoscopic, radiological and histopathological criteria were included between 2006 and 2009. All patients showed previous failure to IFX therapy due to loss of responsiveness (defined as the appearance of symptoms or evidence of disease activity despite the increase in the dose of IFX to $10 \mathrm{mg} / \mathrm{kg}$ and shorter intervals between doses, reduced to 4 weeks), intolerance (defined as the presence of hypersensitivity reactions or adverse events) and lack of initial response to the drug. Prior to treatment with ADA, all patients underwent tuberculin test and chest X-rays.

In 24 out of 25 patients (24/25), an ADA induction dose of 160/80/40 mg was administered and in one case (1/25) 80/40/40 due to malnutrition and low weight. A maintenance dose of $40 \mathrm{mg}$ every two weeks was prescribed, which could be increased to a weekly dose if necessary, this is, if clinical remission was not achieved, if complete closure of the fistulae was not observed or if corticoids were withdrawn. Follow-up duration was 12 months.

The aim was to assess the efficacy of ADA in this group of patients with previous failure to IFX treatments and a retrospective analysis was performed by measuring the Crohn's Disease Activity Index (CDAI), the C-reactive protein (CRP) levels, fistulae closure with clinical confirmation of effusion absence and corticoid withdrawal 0-6-12 months after ADA treatment onset.

All patients signed an informed consent to receive ADA treatment in compassionate use.

Data were processed with the SPSS version 15.0 for Windows. Quantitative variables were expressed as means \pm standard deviation. Qualitative variables were expressed as cohort counts and percentages. Significant differences were considered when $p \leq 0.05$ by using the T-Student tests for quantitative variables.
Table I.

\begin{tabular}{ll}
\hline Basal characteristics & $\begin{array}{l}\text { Patients treated with } \\
\text { adalimumab }(n=25)\end{array}$ \\
\hline Gender & \\
Male & $10(40 \%)$ \\
Female & $15(60 \%)$ \\
Age (years) & $38.3 \pm 12.4$ \\
Disease duration (years) & $10.6 \pm 5.4$ \\
Smoking habit & $3(12 \%)$ \\
Basal CDAl (DS) & $208.1 \pm 78.5$ \\
C reactive protein (mg/L) & $21.9 \pm 26.9$ \\
Disease location & \\
L1 $=$ ileon & $3(12 \%)$ \\
L2 $=$ colon & $8(32 \%)$ \\
L3 = ileo-colonic & $14(56 \%)$ \\
Disease course & \\
B1 = non-stenosing, non-penetrant & $16(64 \%)$ \\
B2 = stenosing & $1(4 \%)$ \\
B3 = perforant & $8(32 \%)$ \\
P = perianal disease & $10(40 \%)$ \\
Previous intestinal surgery & $8(32 \%)$ \\
Concomitant treatment & \\
Corticoids & $15(60 \%)$ \\
Azathioprine & $11(44 \%)$ \\
Methotrexate & $2(8 \%)$ \\
Adalimumab indication & \\
Infliximab efficacy loss & $21(84 \%)$ \\
Infliximab intolerance & $2(8 \%)$ \\
Infliximab unresponsiveness & $1(4 \%)$ \\
Technical difficulties & $1(4 \%)$ \\
\hline & \\
\hline &
\end{tabular}

\section{RESULTS}

Fifteen out of 25 patients $(60 \%)$ were women and 10 $(40 \%)$ were men, with mean age of $38.32 \pm 12.39$ years old and mean duration of CD of $10.64 \pm 5.46$ years. According to the Montreal classification, $12 \%$ of the patients (3/25) present L1-ileal location, 32\% (8/25) L2colonic location, and $56 \%(14 / 25)$ L3-ileocolonic. Furthermore, 11 out of 25 patients $(44 \%)$ had associated fistulizing disease, 10 had perianal disease and one had enterocutaneous disease, included among perforant diseases (Table I).

All patients had received previous treatment with IFX, during an average time of $30 \pm 20.81$ months. Among these, $20(80 \%)$ didn't respond to treatment, $2(8 \%)$ didn't show initial response, adverse events were present in $2(8 \%)$ other patients and in 1 patient $(4 \%)$ we had issues in the canalization of the peripheral intravenous catheter. One of the patients who required drug interruption due to adverse events, presented an anaphylactic reaction during the infusion process, which was solved by i.v. corticoid administration. The other patient presented an acute abdominal pain episode; this patient had a local- 


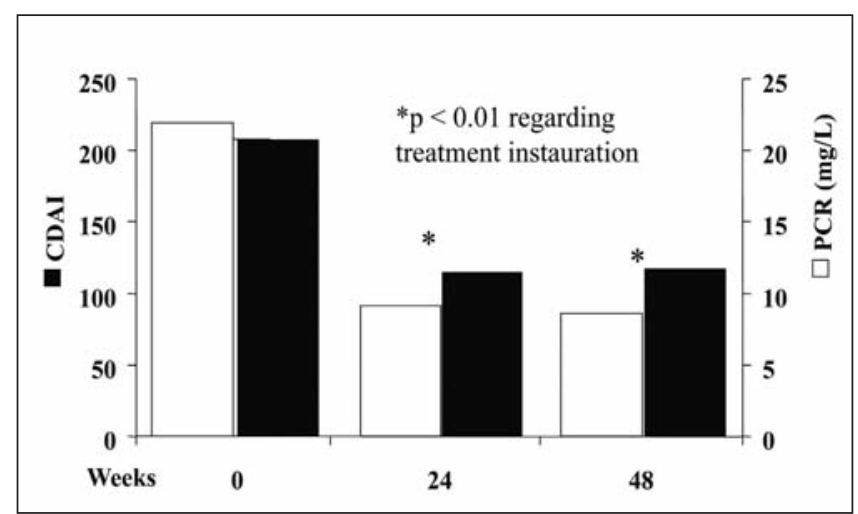

Fig. 1.

ized stenosis on the terminal portion of the ileum, which was diagnosed by small bowel follow through.

During IFX treatment, all patients received prednisone, which had been suspended in $40 \%$ of the patients. Moreover, they had all received azathioprine (23 patients) or methotrexate ( 2 patients). In three cases, azathioprine was suspended due to gastrointestinal discomfort and in two other cases, due to acute pancreatitis. At ADA treatment onset, $60 \%$ of the patients were receiving prednisone, $36 \%$ continued with azathioprine and 12\% were receiving methotrexate.

ADA treatment was responsible for the improvement of disease global activity indexes and parameters. Hence, mean CDAI values were significantly decrease (Fig. 1) from 208, at treatment onset, to 115 after 24 weeks and remained low until the end of the study. Mean CRP concentrations showed a similar significant variation (Fig. 1). Prior to ADA treatment in 5 patients (20\%) CDAI was normal, although 4 of them presented perianal and enterocutaneous fistulae with an important output; in the last case, corticodependence was very high despite IFX treatment. In week 24,18 out of 25 patients $(72 \%)$, and in week 48,15 out of 25 patients $(60 \%)$, showed clinical remission. However, in 18 out of 25 patients (72\%), treatment intervals required to be shortened to one week in order to obtain a sustained response.

In 5 patients (20\%), adverse events were observed, leading to drug withdrawal in 2 cases $(8 \%)$, while in the other $12 \%$ adverse events were mild, such as pain at the point of infusion, headache and febricula in the 12 hours following $\mathrm{ADA}$ administration.

In 4 out of 25 patients (16\%), ADA was suspended before the end of the follow-up period, in 2 of them 6 months after treatment onset due to the lack of response of the fistulizing disease and in another patient, there was a loss of responsiveness and underwent surgical treatment in order to resect an ileocecal stenosis, allowing a further positive control of the disease. In two patients, the drug was suspended due to severe adverse events: in one case an abdominal abscess was reported and further solved by surgery, with a positive control of the disease with immunosuppressants. The other patient developed tuberculous meningitis which responded to tuberculostatic treatment. However, intestinal disease was not controlled in this case and the patient deceased one year later. All patients tolerated ADA administration.

None of the patients with lack of initial response to IFX or adverse reactions to this drug achieved complete remission on week 48; on the other hand 14 of 20 patients $(70 \%)$ with loss of effectiveness to IFX were in a quiescent phase of the disease on week 48. Fifteen of the 25 patients $(60 \%)$ present corticodependence prior to ADA treatment onset. Corticoid suspension was possible in 9 out of 15 patients $(60 \%)$ and in 3 out of 11 (27\%), fistulae closure was achieved during ADA treatment period.

\section{DISCUSSION}

Incomplete response in time or intolerance to IFX treatment are important issues in patients with $\mathrm{CD}$ treated with IFX (3). However, the recent approval of other antiTNF drugs such as certolizumab and ADA has favored research of rescue therapy efficacy in patients with $\mathrm{CD}$ reporting treatment failure to $\operatorname{IFX}(4,7)$.

ADA is a human monoclonal IgG1 antibody which has proven its efficacy in clinical remission induction and maintenance of patients with moderate-severe $\mathrm{CD}$. In our series, induction doses 160/80 were used, based on data from the CLASSIC I study (5), except for one patient in which we used 80/40 doses due to the pronounced malnutrition and low weight of the latter. After 24 and 48 weeks, 72 and $60 \%$ of the patients, respectively, achieved clinical remission in our series. Despite this study presents limitations such as sample size, being an open study or including a heterogeneous group of patients, the results confirm those reported in a similar series recently published in Spain (8). Similarly, they support other data on ADA's efficacy as maintenance treatment versus placebo in controlled studies such as the CLASSIC II (6) and the CHARM studies, in which untreated and patients previously treated with IFX were included. In these series, remission rates at weeks 26 and 54 were 47 and $41 \%$, respectively, without finding significant differences between the employed maintenance doses: $40 \mathrm{mg}$ every one or two weeks, nor between those previously treated with IFX or untreated (9). However, $72 \%$ of our patients required dose intensification. The cause of this remains unknown as preliminary studies (10) have not shown any gene polymorphisms involved in cytokine regulation related to ADA response. It has been suggested that patients with previous failure to IFX present higher levels of resistance to other anti-TNF drugs. However, 77\% (14/18) of the cases may achieve remission in week 48 . These results also suggest that in patients with partial response to standard treatment with ADA, weekly injections could be an effective option, as shown by other studies $(11,12)$. 
Other authors have observed acceptable remission rates even in patients with primary failure to IFX such as in the GAIN study (13-15).

Regarding fistulizing disease, complete closure was achieved in $27 \%$ of the cases in week 48 , which is in agreement with data obtained in the CHARM study, according to which in week $56,30 \%$ of the patients receiving ADA present complete closure of the fistulae in comparison to the placebo group, in which $13 \%$ achieved this aim. In the CLASSIC study remission rates of fistulizing disease after 4 weeks of treatment were 20 and $23 \%$, respectively (16), as well as in the study published by Hinojosa et al. (uncontrolled study). In our series, steroid treatment withdrawal was achieved in $60 \%$ of the corticodependent subjects in week 48 , which is a higher proportion of patients than that reported in the CHARM study (29\%). On the other hand, in the CLASSIC II study, steroid withdrawal was possible in most patients treated with ADA, although the causes of these differences remain unknown. During ADA treatment, $20 \%$ of the patients present adverse events, most of them mild and well-tolerated. However, two patients (8), required treatment withdrawal due to the appearance of severe infectious events, an abdominal abscess and tuberculous meningitis, despite screening for tuberculosis prior to ADA (and IFX) treatment. Hypersensitivity events were not reported.

In conclusion, adalimumab is an effective treatment in patients with $\mathrm{CD}$ and failure to previous treatment when achieving clinical remission induction and maintenance, although in this subgroup of patients, therapy intensification is frequently required.

\section{REFERENCES}

1. Rutgeerts P, Van Assche G, Vermiere S. Optimizing anti-TNF treatment in inflammatory bowel disease. Gastroenterology 2004;126: 1593-610.

2. Mease PJ. Adalimumab in the treatment of arthritis. Ther Clin Risk Manag 2007;3(1):133-48.

3. Peyrin-biroulet L, Laclotte C, Bigard MA. Adalimumab maintenance therapy for Crohn s disease with intolerance or lost response to infli- ximab: an open-label study. Aliment Pharmacol Ther 2007;25:67580.

4. Seiderer J, Brand S, Dambacher J, Pfenning S, Jürgens M, Göke B, et al. Adalimumab in patients with Crohn s disease -safety and efficacy in an open-label single centre study. Aliment Pharmacol Ther 2007; 25:787-96.

5. Hanauer SB, Sandborn WJ, Rutgeerts P, Fedorak RN, Lukas M, McIntosh D, et al. Human anti-tumor necrosis factor monoclonal antibody (adalimumab) in Crohn s disease: the CLASSIC-I trial. Gastroenterology 2006;130:323-33.

6. Sandborn WJ, Hanauer SB, Rutgeerts P, Fedorak RN, Lukas M, MacIntosh DG, et al. Adalimumab for maintenance treatment of Crohn s disease: results of the CLASSIC II trial. Gut 2007;56:1232-9.

7. Allez M, Vermiere S, Mozziconacci N, Michetti P, Laharie D, Louis E, et al. Efficacy and safety o a third anti-TNF monoclonal antibody in Crohn s disease after failure of two other anti-TNF. Aliment Pharmacol Ther 2009;26.

8. López Palacios N, Mendoza JL, Taxonera C, Lana R, Fuentes Ferrer M, Díaz-Rubio M. Adalimumab induction and maintenance therapy for Crohn s disease. An open-label study. Rev Esp Enferm Dig 2008; 100:676-81.

9. Colombel JF, Sandborn WJ, Rutgeerts P, Enns R, Hanuer SB, Panaccione $\mathrm{R}$, et al. Adalimumab form maintenance of clinical response and remission in patients with Crohn's disease: The CHARM trial. Gastroenterology 2007;132:52-65.

10. Barreiro-de Acosta M, Ouburg S, Morré SA, Crusius JB, Lorenzo A, Potel J, et al. NOD2, CD14 and TLR4 mutations do not influence response to adalimumab in patients with Crohn $\mathrm{s}$ disease: a preliminary report. Rev Esp Enferm Dig 2010;102:591-5.

11. Schiff MH, Burmester GR, Kent JD, Pangan AL, Kupper H, Fitzpatrick SB, et al. Safety analyses of adalimumab (HUMIRA) in global clinical trials and US postmarketing surveillance of patients with rheumatoid arthritis. Ann Rheum Dis 2006;65:889-94.

12. Trinder MW, Lawrance IC. Efficacy of adalimumab for the management of inflammatory bowel disease in the clinical setting. J Gastroenterol Hepatol 2009;24:1252-7.

13. Sandborn WJ, Rutgeerts P, Enns R, Hanauer SB, Colombel JF, Panaccione R, et al. Adalimumab induction therapy for crohn disease previously treated with infliximab. A randomized trial. Ann Intern Med 2007;146:829-38.

14. Ho GT, Smith L, Aitken S, Lee HM, Ting T, Fennell J, et al. The use of adalimumab in the management of refractory Crohn s disease. Aliment Pharmacol Ther 2007;27:308-15.

15. Sandborn WJ, Hanauer S, Loftus Ev Jr, Tremaine WJ, Kane S, Cohen R, et al. An open-label study of the human anti-TNF monoclonal antibody adalimumab in subjects with prior loss of response or intolerance to infliximab for Crohn s disease. Am J Gastroenterol 2004; 99:1984-9

16. Hinojosa J, Gomollón F, Garcia S, Bastida G, Cabriada JL, Saro C, et al. Efficacy and safety of short-term adalimumab treatment in patients with active Crohn s disease who lost response or showed intolerance to infliximab: a prospective, open-label, multicentre trial. Aliment Pharmacol Ther 2007;25:409-18. 